Z Rheumatol 2020 $\cdot 79: 294-303$ https://doi.org/10.1007/s00393-019-00724-y Online publiziert: 21. November 2019 (c) Der/die Autor(en) 2019

\section{Redaktion}

U. Müller-Ladner, Bad Nauheim

U. Lange, Bad Nauheim

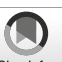

\author{
A. Prasse ${ }^{1} \cdot$ F. Bonella ${ }^{2}$ U. Müller-Ladner ${ }^{3} \cdot$ T. Witte $^{4} \cdot$ N. Hunzelmann ${ }^{5} \cdot$ J. Distler $^{6}$ \\ ${ }^{1}$ Klinik für Pneumologie, Medizinische Hochschule Hannover, Hannover, Deutschland \\ ${ }^{2}$ Ruhrlandklinik, Klinik für Pneumologie, Universitätsmedizin Essen, Essen, Deutschland \\ ${ }^{3}$ Abteilung für Rheumatologie und Klinische Immunologie, Justus-Liebig-Universität Gießen, Campus \\ Kerckhoff, Bad Nauheim, Deutschland \\ ${ }^{4}$ Klinik für Immunologie und Rheumatologie, Medizinische Hochschule Hannover, Hannover, Deutschland \\ ${ }^{5}$ Klinik und Poliklinik für Dermatologie und Venerologie, Uniklinik Köln, Köln, Deutschland \\ ${ }^{6}$ Klinik für Rheumatologie und Immunologie, Universitätsklinikum Erlangen, Erlangen, Deutschland
}

\title{
Therapie der systemischen Sklerose-assoziierten interstitiellen Lungenerkrankung
}

zeichnend und zählt zu den häufigsten und nach dem Raynaud-Phänomen oft auch ersten Symptomen der Krankheit. Je nach Ausmaß der Organbeteiligung kann es zum Auftreten weiterer Krankheitszeichen wie etwa Dysphagie, Reflux, Arthritis oder digitalen Ulzerationen kommen [6]. Tritt die SSc ohne klinisch nachweisbare Hautbeteiligung in Erscheinung, so spricht man von einer SSc sine Scleroderma (ssSSc).

\section{Lungenbeteiligung}

Pulmonale Manifestationen zählen $\mathrm{zu}$ den häufigsten Organbeteiligungen und treten bei bis zu $90 \%$ der SSc-Patienten auf [13]. Eine klinisch relevante SScassoziierte interstitielle Lungenerkrankung (SSc-ILD) findet sich bei nahezu der Hälfe aller Betroffenen mit SSc und zählt zu den häufigsten Todesursachen der Erkrankung [42, 46]. Eine ILD als Erstmanifestation der SSc wurde bei bis zu $15 \%$ der Fälle berichtet.

Ein Befall der Lunge in Form einer SSc-ILD äußert sich oftmals durch zunehmende Belastungsdyspnoe, die häufig durch Reizhusten und unspezifische Symptome wie Müdigkeit oder Gewichtsabnahme begleitet wird. Fast immer kommt es bei SSc-Patienten zudem im Rahmen einer Refluxerkrankung zu rezidivierenden (Mikro-)Aspirationen, die die Ausbildung und Progression der ILD begünstigen [34]. Im klinischen
Alltag fällt die Abgrenzung von aspirationsbedingten Lungenschädigungen und Fibroseprogress oft schwer. Differenzialdiagnostisch bedeutsam bei Belastungsdyspnoe im Kontext einer SSc ist zudem der Ausschluss anderer SScManifestationen und Komorbiditäten wie der pulmonalen Hypertonie und der myokardialen Beteiligung, aber auch einer koronaren Herzkrankheit. Neue Daten zeigen auch, dass eine schnelle Hautprogression mit einer sich parallel entwickelnden Progression der Lungenfibrose assoziiert ist [51].

\section{Diagnostik der Erkrankung}

Die im Jahr 2013 vorgestellten American College of Rheumatology (ACR)/ European League Against Rheumatism(EULAR)-Klassifikationskriterien

(- Tab. 1) erlauben es, eine SSc mit hoher Sensitivität zu klassifizieren [18]. Der Nachweis spezieller antinukleärer Antikörper(ANA)-Profile gibt dabei häufig Aufschlüsse zur vorliegenden Form der Erkrankung sowie zu deren Prognose (• Tab. 2).

Die Früherkennung der Lungenfibrose (SSc-ILD) hat eine hohe klinische und therapeutische Relevanz. Wichtiger Teil der Diagnostik ist hierbei die Lungenauskultation. Die SSc-ILD manifestiert sich fast immer in den dorsobasalen Anteilen der Lunge und erzeugt bereits im Frühstadium das charakteristische 
Tab. 1 ACR/EULAR-Kriterien für die Klassifizierung der SSc [18]

Kriterium

Hautverdickung der Finger beider

Hände, die sich proximal zu den Meta-

karpophalangealgelenken erstrecken

(ausreichendes Kriterium)

Hautverdickung der Finger (höhere

Punktzahl zählt)

Läsionen an den Fingerspitzen

(höhere Punktzahl zählt)

Teleangiektasie

Abnormale Nagelfalzkapillaren

Pulmonale Hypertonie und/oder interstitielle Lungenerkrankung

(maximale Punktzahl ist 2)

Raynaud-Syndrom

Autoantikörper der systemischen

Sklerose (maximale Punktzahl ist 3)

\section{Gewichtung}

9

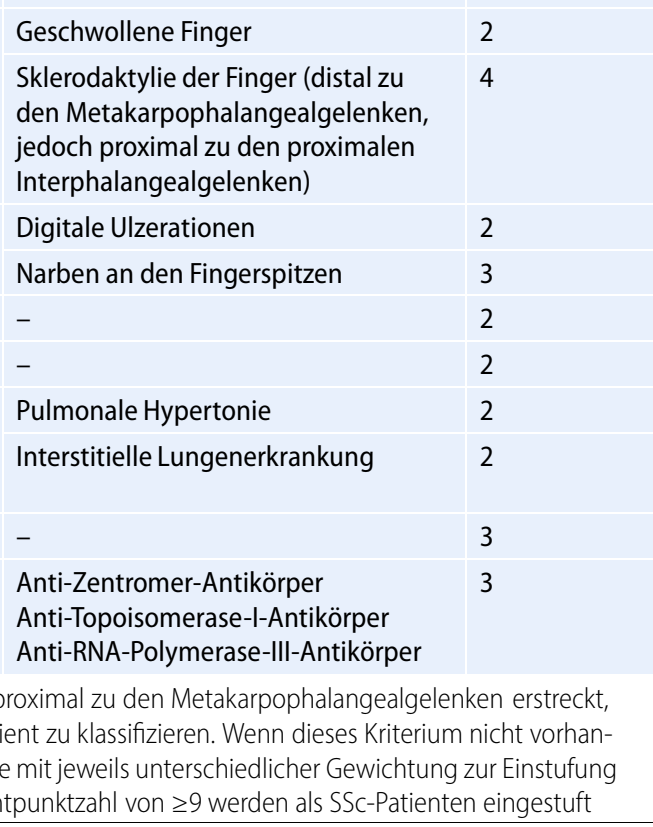

Tab. 2 Autoantikörper bei systemischer Sklerose (SSC)

\begin{tabular}{|c|c|c|}
\hline Autoantikörper & Erkrankungsform & Organbeteiligung \\
\hline $\begin{array}{l}\text { Anti-Topoisomerase-I-Antikörper } \\
\text { (Anti-Scl-70) }[2,17,32]\end{array}$ & Diffus kutane SSC & $\begin{array}{l}\text { Erhöhtes Risiko für: } \\
\text { ILD/Lungenfibrose } \\
\text { Nierenbeteiligung } \\
\text { Herzbeteiligung } \\
\text { Pulmonale Hypertonie } \\
\text { FVC Reduktion }\end{array}$ \\
\hline \multirow[t]{2}{*}{ Anti-Zentromer-Antikörper [10, 32] } & \multirow[t]{2}{*}{ Limitiert kutane SSC } & $\begin{array}{l}\text { Erhöhtes Risiko für: } \\
\text { Pulmonale Hypertonie }\end{array}$ \\
\hline & & $\begin{array}{l}\text { Niedriges Risiko für: } \\
\text { ILD/Lungenfibrose } \\
\text { Nierenbeteiligung }\end{array}$ \\
\hline $\begin{array}{l}\text { Anti-RNA-Polymerase-III-Antikörper [10, } \\
23,47]\end{array}$ & Diffus kutane SSC & $\begin{array}{l}\text { Erhöhtes Risiko für: } \\
\text { Nierenbeteiligung } \\
\text { Pulmonale Hypertonie } \\
\text { Paraneoplasie }\end{array}$ \\
\hline Anti-PM-Scl-Antikörper [17] & Limitiert kutane SSC & $\begin{array}{l}\text { Erhöhtes Risiko für: } \\
\text { Myositis }\end{array}$ \\
\hline Anti-U3-RNP-Antikörper (Fibrillarin) [17] & Diffus kutane SSC & $\begin{array}{l}\text { Erhöhtes Risiko für: } \\
\text { Organbeteiligung/ILD } \\
\text { Nierenbeteiligung } \\
\text { Pulmonale Hypertonie }\end{array}$ \\
\hline
\end{tabular}

inspiratorische Knisterrasseln. Zusätzlich sollte eine Lungenfunktionsprüfung erfolgen. Hierbei weisen ca. 40-75\% der Patienten mit SSc-ILD eine restriktive Ventilationsstörung mit Einschränkungen von Gesamtlungenvolumen (TLC), forcierter Vitalkapazität (FVC) und Diffusionskapazität (DLCO) auf. Besonders die Dynamik von Veränderungen in der Lungenfunktion spielt bei der Diagnostik der ILD eine entscheidende Rolle. Aus Expertensicht besteht Konsens, dass auch bei normalen Lungenfunktionswerten eine hochauflösende Computertomographie (HRCT) bei auskultatorischem Knisterrasseln als Screeninguntersuchung auf eine SSc-ILD befürwortet werden sollte [35]. Hierbei kann auch die für die SSc pathognomonische Dilatation des Ösophagus nachgewiesen werden. Im HRCT wird eine Lungenbeteiligung auch im Frühstadium sehr gut erkannt. Das am häufigsten $(80 \%)$ auftretende radiologische Muster ist hierbei die nichtspezifische interstitielle Pneumonie (NSIP) (• Abb. 1).

Seltener $(20 \%)$ findet sich bei SScILD-Patienten die gewöhnliche interstitielle Pneumonie (UIP) (• Abb. 2). Das UIP-Muster tritt vorzugsweise bei Männern mit positiver Raucheranamnese und Staubbelastung am Arbeitsplatz auf [22]. Dieselben Risikofaktoren sind auch für die idiopathische Lungenfibrose, die strikt mit einem UIP-Muster einhergeht, beschrieben worden [34].

Diagnostisch schwierig ist die Einschätzung der Progression der ILD. Bei manchen Patienten kann die SSc-ILD über Jahrzehnte stabil bleiben, während andere einen rasch progredienten Verlauf oder sogar eine akute Exazerbation aufweisen [15]. Eine engmaschige Betreuung und Überwachung von SSc-ILDPatienten in speziell ausgewiesenen Zentren des Deutschen Netzwerks für Systemische Sklerose e. V. (DNSS) ist daher sinnvoll. Zu den wichtigsten Risikofaktoren einer progredienten SSc-ILD zählen ein hohes Ausmaß interstitieller Veränderungen im HRCT ( $>20 \%$ des gesamten Lungenvolumens) sowie eine deutlich verminderte forcierte Vitalkapazität (FVC <70\%) [4, 16]. Regelmäßige Lungenfunktionsuntersuchungen sollten daher mindestens jährlich, bei Patienten mit besonders progressivem Krankheitsbild auch öfter erfolgen. Einige Zentren evaluieren als weitere potenzielle Indikatoren für Krankheitsaktivität zudem Serumbiomarker und die BAL-Differenzialzytologie [38].

Aufgrund der Variabilität der Krankheitsverläufe erfordert die Diagnose einer SSc-ILD ein umfangreiches Expertenwissen, das auf der Kompetenz in der Betreuung der SSc erfahrener Kliniker aus verschiedenen Fachdisziplinen auf- 
baut [1]. Eine multidisziplinäre Fachkonferenz (oder ILD Board) gilt zur Diagnostik fibrotischer ILDs mittlerweile als Goldstandard [1].

Das Ziel des vorliegenden Reviews besteht darin, einen Überblick über bestehende und mögliche zukünftige Behandlungsansätze der SSc-ILD zu geben. Es wurde eine Literaturrecherche durchgeführt, die sich auf die relevantesten Beiträge der zwischen 2010 und 2018 veröffentlichten, medizinischen Literatur konzentriert.

\section{Allgemeine Behandlungs- ansätze}

Die Behandlung der SSc ist komplex und oft mit hohen gesundheitsökonomischen Kosten verbunden. Insbesondere bei Vorliegen einer SSc mit pulmonaler Beteiligung bedarf es einer frühzeitigen Behandlung, um ein Fortscheiten der Erkrankung der Lunge zu verzögern. Neben einer medikamentösen Behandlung sollten auch nichtpharmakologische Maßnahmen genutzt werden. Ansätze wie Physiotherapie, Sauerstofftherapie, Pneumokokken- und Grippeschutzimpfungen oder der Einsatz gastroenterologischer Maßnahmen (Einsatz von Protonenpumpenhemmern, nächtliche Oberkörperhochlagerung, kleine, nicht zu späte Mahlzeiten) können dabei helfen, den Krankheitsverlauf günstig zu beeinflussen bzw. Symptome zu verbessern.

Eine weitere Therapieoption, die bei jüngeren Patienten mit weit vorangeschrittener SSc-ILD immer in Erwägung gezogen werden sollte, ist die Möglichkeit einer Lungentransplantation. Aufgrund der häufig vorhandenen erheblichen Komorbiditäten ist dies jedoch bei SScPatienten oft nicht realisierbar.

\section{Aktuelle Leitlinienempfehlungen}

Die EULAR-Empfehlungen zur Behandlung der systemischen Sklerose wurden im Jahr 2017 unter Berücksichtigung neu verfügbarer Pharmakotherapien von einer europäischen Task Force aktualisiert (• Tab. 3; [29]).

Für die Behandlung bei SSc-ILD empfehlen die EULAR-Richtlinien eine

Z Rheumatol 2020 · 79:294-303 https://doi.org/10.1007/s00393-019-00724-y

(c) Der/die Autor(en) 2019

\section{A. Prasse $\cdot$ F. Bonella $\cdot$ U. Müller-Ladner $\cdot$ T. Witte $\cdot$ N. Hunzelmann $\cdot$ J. Distler Therapie der systemischen Sklerose-assoziierten interstitiellen Lungenerkrankung}

\section{Zusammenfassung}

Hintergrund. Die systemische Sklerose (SSc) ist eine fibrosierende Autoimmunerkrankung aus der Gruppe der Kollagenosen. Neben der Hautfibrose zählt eine Beteiligung der Lunge, insbesondere in Form einer interstitiellen Lungenerkrankung (ILD), zu den häufigsten und schwersten Organmanifestationen der SSc. Die Krankheit geht insbesondere bei progressiver ILD mit einer erheblichen Morbidität und Mortalität einher. In den letzten 5 Jahren wurden in zahlreichen klinischen Studien neue Behandlungskonzepte für die SSc-ILD untersucht.

Material und Methoden. Im Rahmen dieses Reviews erfolgte eine Literaturrecherche mittels PubMed, die sich auf die relevantesten Beiträge der bis Ende 2018 veröffentlichten medizinischen Literatur mit den Schlagwörtern "SSc" und "Treatment" konzentrierte.

Ergebnisse. Die Therapie der SSc-ILD hat sich in den letzten Jahren aufgrund der Ergebnisse zahlreicher klinischer Studien gewandelt. Die aktualisierten Leitlinien der Europäischen
Rheumatologengesellschaft (EULAR) empfehlen den Einsatz von Cyclophosphamid oder einer hämatopoetischen Stammzelltransplantation. Daten zur positiven Beeinflussung der SSc-ILD liegen auch für Mycophenolat, Tocilizumab und Anabasum vor. Aufgrund der pathophysiologischen Gemeinsamkeiten zur idiopathischen Lungenfibrose wird derzeit der Einsatz der antifibrotischen Wirkstoffe Nintedanib und Pirfenidon in randomisierten, multizentrischen klinischen Studien getestet und könnte eine zusätzliche, vielversprechende Therapiestrategie sein. Schlussfolgerung. Innovative therapeutische Perspektiven für die SSc-ILD sind durch Erfolg versprechende Ansätze aktueller Arzneimittelstudien gegeben und könnten in Zukunft die Prognose von Betroffenen merklich verbessern.

Schlüsselwörter

Systemische Sklerose - Bindegewebserkrankung · Organbeteiligung · Interstitielle Lungenerkrankung · Therapie

\section{Treatment of systemic sclerosis-associated interstitial lung disease}

\section{Abstract}

Background. Systemic sclerosis (SSC) is a fibrosing autoimmune disease of the connective tissue. In addition to skin fibrosis, pulmonary involvement and interstitial lung disease (ILD) in particular are the most common and severe manifestations of SSc. The disease is associated with a substantial risk of morbidity and mortality, especially in progressive ILD. In the last 5 years new treatment concepts for SSc-ILD have been investigated in numerous clinical studies. Material and methods. This review is based on a literature search in PubMed, focusing on the most relevant papers published up to the end of 2018 with the keywords "SSc" and "treatment".

Results. The treatment of SSc-ILD has changed over the last few years due to the results of many clinical studies. The updated guidelines of the European League Against Rheumatism (EULAR) recommend the use of cyclophosphamide or hematopoietic stem cell transplantation. Data for a positive influence on SSC-ILD are also available for mycophenolate, tocilizumab and anabasum. Because of the pathophysiological similarities to idiopathic pulmonary fibrosis, the use of the antifibrotic agents nintedanib and pirfenidone is currently being investigated in randomized, multicenter clinical trials and could be a novel and promising therapeutic strategy.

Conclusion. Current drug studies may provide innovative therapeutic perspectives for SScILD and could significantly improve the prognosis of affected patients in the future.

\section{Keywords}

Systemic sclerosis - Connective tissue disease . Organ involvement $\cdot$ Interstitial lung disease . Therapy 


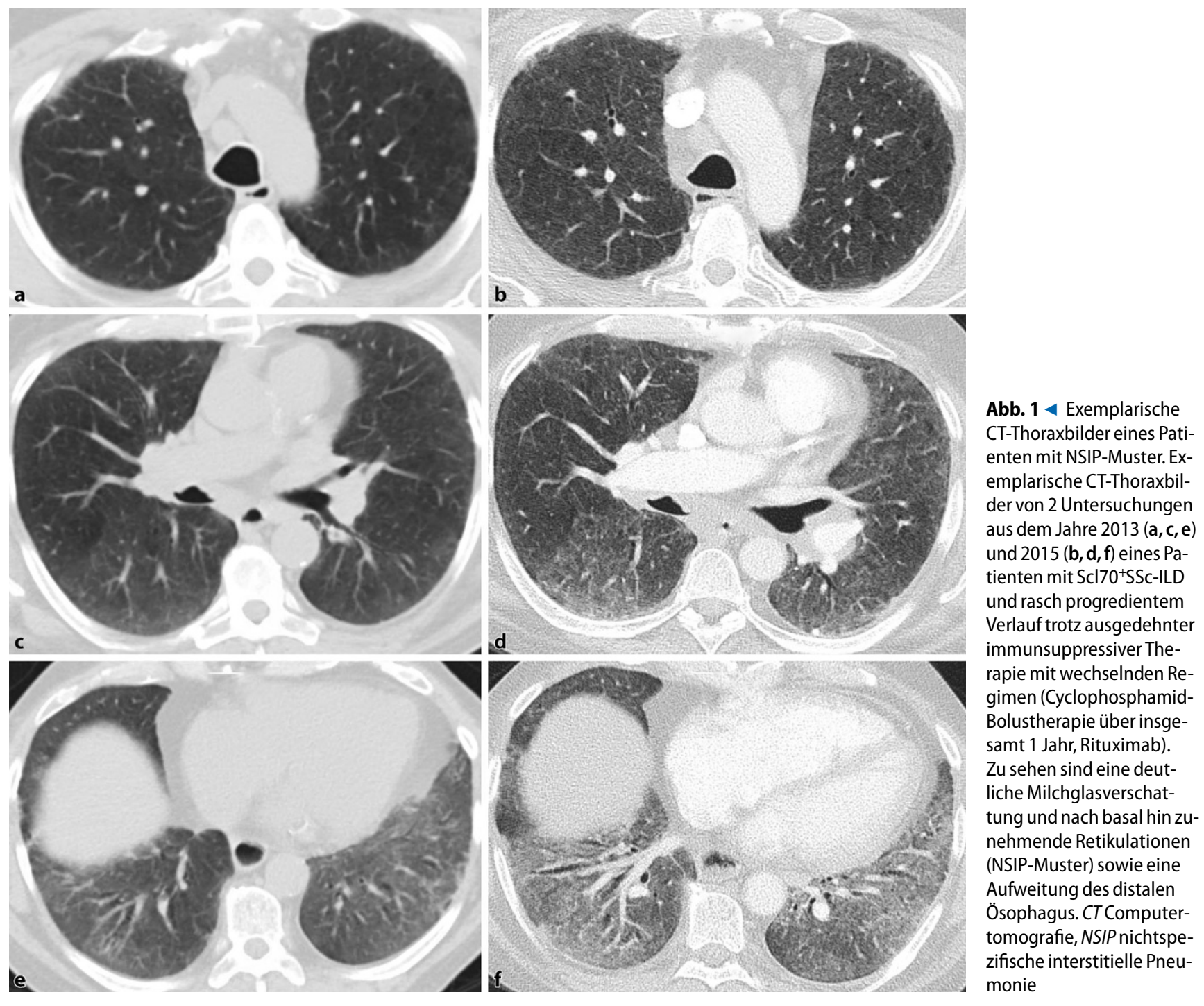

Therapie mit dem Zytostatikum Cyclophosphamid (CYC), die angesichts der Ergebnisse zweier hochqualitativer RCTs gestützt wird [19, 29, 44]. So zeigte die Scleroderma Lung Study (SLS I) einen signifikant positiven Effekt von oral verabreichtem CYC auf Lungenfunktion, Dyspnoe, Verdickung der Haut und gesundheitsbezogene Lebensqualität [44]. In einer zweiten Studie führte eine intravenös verabreichte CYC-Behandlung $\mathrm{zu}$ einer Verbesserung der FVC um 4,1\%, aber verfehlte damit die Schwelle zur statistischen Signifikanz $(p=0,08)$ [19].

In Anbetracht der Studienergebnisse beider RCTs sollte Cyclophosphamid trotz bekannter, geringer Toxizität bei der Behandlung der SSc-ILD insbesondere bei Patienten mit rasch progredienter ILD in Betracht gezogen werden. Die beglei- tende Antibiotikagabe zur Pneumocystisjirovecii-Pneumonie( $\mathrm{Pj} \mathrm{P})$-Prophylaxe ist bei CYC-Behandlung zwingend erforderlich [39].

Neben der Behandlung mit CYC wird in den EULAR-Richtlinien eine hämatopoetische Stammzelltransplantation (HSCT) zur Therapie ausgewählter Patienten mit schnell fortschreitender SSc und Risiko für Organversagen empfohlen [29].

SCOT, eine randomisierte klinische Studie, verglich die potenziellen Vorteile einer HSCT mit denen einer hoch dosierten, 12-monatigen CYC-Therapie bei Patienten mit schweren SScVerlaufsformen. Hierbei erzielte die myeloablative autologe hämatopoetische Stammzelltransplantation bessere Langzeitergebnisse als die CYC-Therapie, einschließlich verbessertem ereignisfreiem Überleben und Gesamtüberleben [43].

In der europäischen ASTIS-Studie verbesserte eine HSCT signifikant das langfristige ereignisfreie Überleben gegenüber einer CYC-Pulstherapie und führte nach 2 Jahren $\mathrm{zu}$ signifikanten Verbesserungen von mRSS, FVC und TLC [30]. Angesichts des hohen Risikos von behandlungsbedingten Nebenwirkungen und Mortalität ist eine sorgfältige Auswahl der Patienten für diese Art der Behandlung von entscheidender Bedeutung [29]. 

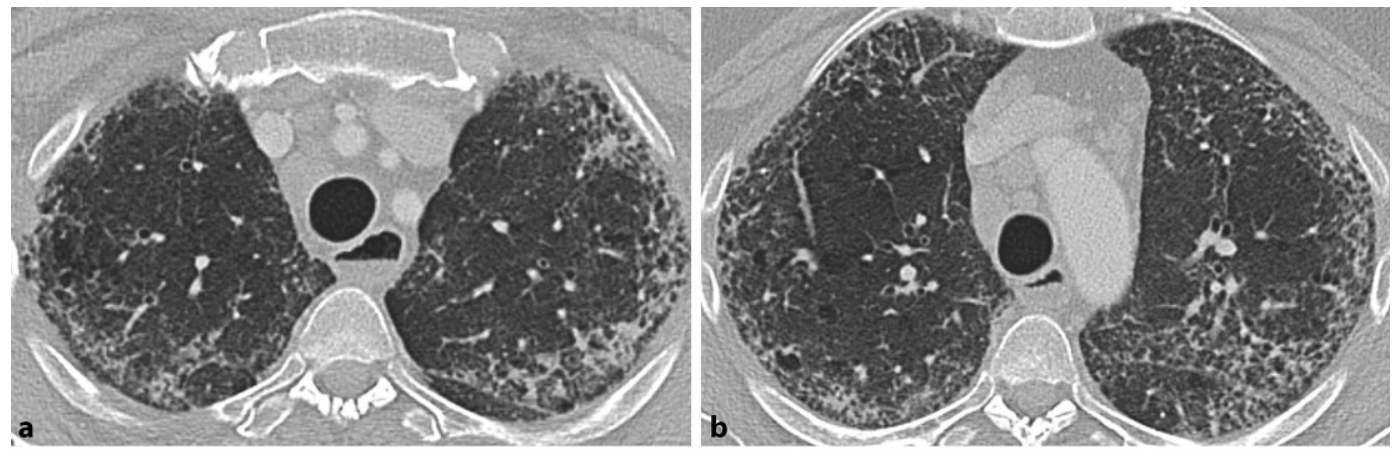

Abb. $2 \triangleleft$ Exemplarische CT-Thoraxbilder eines $\mathrm{Pa}$ tienten mit UIP-Muster. (a-f) CT-Thoraxaufnahmen
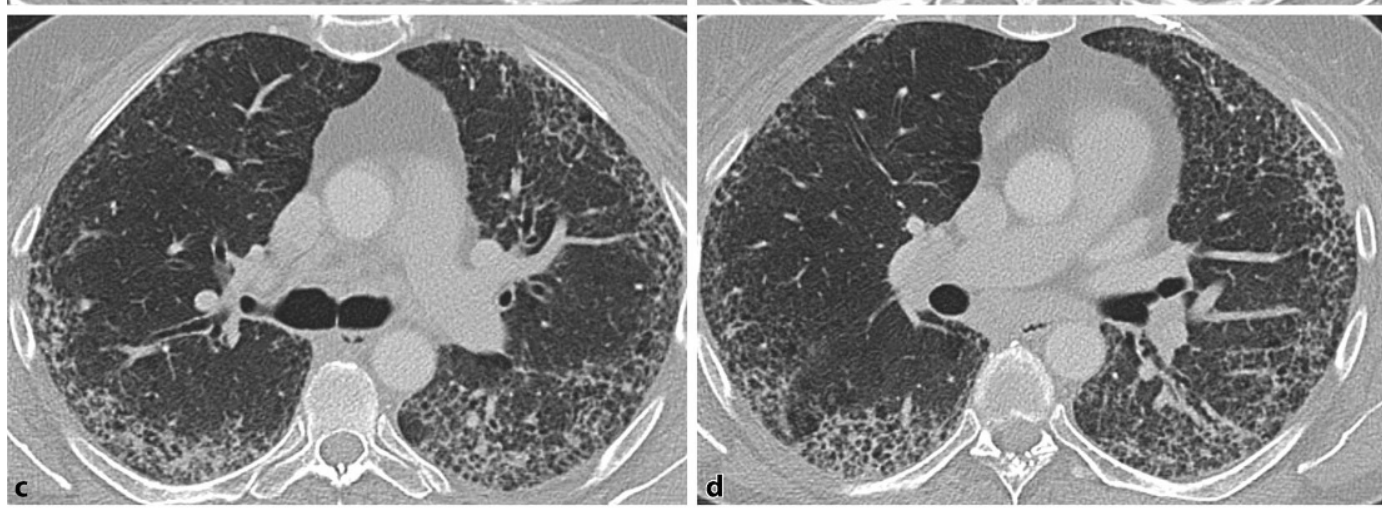
einer 56-jährigen Patientin bei Erstdiagnose einer Scl70+ SSc und langjährigem, weiterhin aktivem Zigarettenrauchen (30 py). Es ist eine subpleurale und dorsobasale Betonung des Verschattungsmusters festzustellen, welches vorwiegend aus Honigwabenzysten und Retikulationen im Sinne eines ausgeprägten, definitiven UIP-Musters besteht. Zudem finden sich eine diffuse Milch-
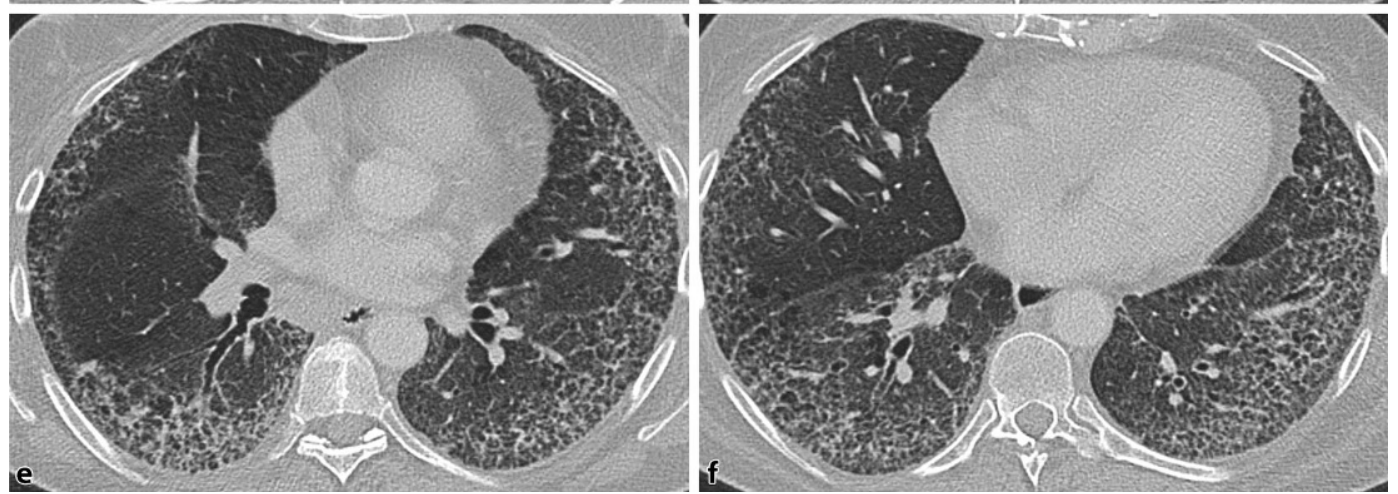
glasverschattung und eine deutliche Aufweitung des Ösophagus mit Flüssigkeitsspiegel. Die Patientin wies bei Erstvorstellung eine schwere respiratorische Partialinsuffizienz mit einem $\mathrm{O}_{2}$-Bedarf von $31 \mathrm{O}_{2} / \mathrm{min}$ auf und verstarb 6 Monate später. CT Computertomografie, $p y \mathrm{~Pa}$ ckungsjahre, UIP gewöhnliche interstitielle Pneumonie

\section{Weitere medikamentöse Therapien}

\section{Steroide}

Bei Betroffenen mit SSc-ILD war eine Therapie mit Kortikosteroiden aufgrund eines Mangels an alternativen Behandlungsoptionen lange Zeit eine häufig verwendete Therapieoption [48]. Der Einsatz von Kortikosteroiden in der Behandlung der ILD ist jedoch aufgrund einer unzureichenden Datenlage bei SSc-ILD sowie des erhöhten Risikos einer renalen Krise besonders bei Prednison-Dosierungen über $15 \mathrm{mg} / \mathrm{Tag}$ nur in Ausnahmen, etwa bei gleichzeitigem Auftreten von Arthritis oder Myositis, indiziert und sollte mit möglichst geringen Dosen durchgeführt werden [48].

\section{Azathioprin}

Azathioprin (AZA), ein Wirkstoff aus der Gruppe der nichtselektiven Immunsuppressiva, wurde in einer randomisierten Studie mit Cyclophosphamid (CYC) als Erstlinientherapie bei SSc-ILD verglichen [31]. Während CYC die FVCund DLCO-Werte stabilisierte, kam es unter AZA-Gabe zu einer signifikanten Verschlechterung von FVC und DLCO. Daher wird Azathioprin nicht als Erstlinientherapieoption bei SSc-ILD betrachtet [31].

\section{Mycophenolat-Mofetil}

Mycophenolat-Mofetil (MMF) ist ein Immunsuppressivum zur Inhibition der Lymphozytenproliferation. Die Scleroderma Lung Study II (SLS II) verglich den Einsatz von MMF mit oral verabreich- tem CYC bei Patienten mit SSc-ILD [45]. Nach einer Studiendauer von 2 Jahren wurden bei beiden Behandlungsgruppen signifikante Verbesserungen in Bezug auf FVC und Mortalität festgestellt. Das Verträglichkeitsprofil von MMF war jedoch verbessert [45]. MMF wird aufgrund dieser Daten von vielen Zentren als Off-label-Use zur Langzeittherapie der SSc-ILD eingesetzt. In der vor Kurzem publizierten SENSCIS ${ }^{\circledast}$-Studie wurde ein positiver Effekt der MycophenolatTherapie auf den 1-Jahres-FVC-Verlauf an einer Kohorte von behandelten 576 Patienten eindrucksvoll belegt. 


\begin{tabular}{|c|c|}
\hline Kategorie & $\begin{array}{l}\text { EULAR-Empfehlungen } \\
2017\end{array}$ \\
\hline $\begin{array}{l}\text { Raynaud-Syndrom } \\
\text { (RP) }\end{array}$ & $\begin{array}{l}\text { Kalziumantagonisten } \\
\text { (DHP-Typ) } \\
\text { i.v. Iloprost } \\
\text { PDE-5-Inhibitoren } \\
\text { Fluoxetin }\end{array}$ \\
\hline $\begin{array}{l}\text { Digitale Ulzeratio- } \\
\text { nen (DU) }\end{array}$ & $\begin{array}{l}\text { i.v. Iloprost } \\
\text { PDE-5-Inhibitoren } \\
\text { Bosentan }\end{array}$ \\
\hline $\begin{array}{l}\text { Pulmonale Hyper- } \\
\text { tonie (PH) }\end{array}$ & $\begin{array}{l}\text { ERA (Ambrisentan, } \\
\text { Bosentan, Macitentan) } \\
\text { PDE-5-Inhibitoren } \\
\text { (Sildenafil, Tadalafil) } \\
\text { Riociguat } \\
\text { i.v. Epoprostenol } \\
\text { Andere Prostazyk- } \\
\text { linanaloga (Iloprost, } \\
\text { Treprostinil) } \\
\text { Selexipag }\end{array}$ \\
\hline Haut/Lunge & $\begin{array}{l}\text { MTX (Haut) } \\
\text { Cyclophosphamid } \\
\text { (ILD) } \\
\text { HSCT }\end{array}$ \\
\hline Renale Krise (SRC) & ACE-Hemmer \\
\hline $\begin{array}{l}\text { Gastrointestinal- } \\
\text { trakt (GI) }\end{array}$ & $\begin{array}{l}\text { Protonenpumpen- } \\
\text { inhibitoren } \\
\text { Prokinetika } \\
\text { Antibiotika }\end{array}$ \\
\hline
\end{tabular}

\section{Zukünftige Therapieansätze in klinischen Studien}

Einige vielversprechende Behandlungsansätze wurden oder werden aktuell in klinischen Studien getestet und könnten die Therapiemöglichkeiten der SSc-ILD in Zukunft maßgeblich erweitern, aktuell handelt es sich hierbei allerdings noch um Off-Label-Behandlungen.

\section{Tocilizumab}

Tocilizumab (TCZ) ist ein humanisierter monoklonaler Interleukin-6-Rezeptor-Antikörper (IgG1), der sich bereits als wirksame Therapie für verschiedene Autoimmunerkrankungen erwiesen hat.

Die Phase-II-Studie FaSScinate verglich die Wirksamkeit und Sicherheit von $162 \mathrm{mg}$ subkutan verabreichtem Tocilizumab/Woche mit Placebo zur Behandlung von Patienten mit früher dcSSc mit und ohne ILD [25]. Tocilizumab war mit einer Stabilisierung der FVC-Werte sowie nichtsignifikanten Verbesserungen der Hautbeteiligung assoziiert [25].

Erste Ergebnisse der Phase-III-Studie focuSSced unterstützen die präventive Wirkung von Tocilizumab auf den Verlust der FVC [26].

\section{Anabasum}

Eine weitere in klinischer Entwicklung befindliche Behandlungsoption der SSc stellt die Therapie mit Anabasum, einem Cannabinoid-Rezeptor-Typ-2-Agonist, dar.

Eine doppelblinde Phase-II-Studie untersuchte Wirksamkeit und Sicherheit von Anabasum bei Patienten mit diffuser SSc und einer Krankheitsdauer von bis zu 6 Jahren [41]. Anabasum führte im Vergleich zu Placebo zu einer signifikanten Verbesserung des ACR Composite Response Index in Systemic Sclerosis (CRISS)-Scores einschließlich Reduktion des FVC-Verlustes und war mit einer guten Verträglichkeit assoziiert [41].

RESOLVE-1, eine placebokontrollierte Phase-III-Studie zur Beurteilung der Wirksamkeit und Sicherheit von Anabasum bei SSc-ILD, wird derzeit durchgeführt. Erste Ergebnisse dieser Studie werden für 2020 erwartet.

\section{Abatacept}

Abatacept, ein CTLA4-Fusionsprotein, das kostimulatorische Signalwege von antigenpräsentierenden Zellen reguliert und somit die T-Zell-Aktivierung beeinträchtigt, wird bereits zur Therapie der rheumatoiden Arthritis eingesetzt. ASSET, eine randomisierte, placebokontrollierte Phase-II-Studie, untersuchte Wirksamkeit und Sicherheit von Abatacept bei Patienten mit diffuser SSc. Obwohl der primäre Endpunkt deutlich verfehlt wurde, führte Abatacept jedoch im Vergleich zu Placebo zu einer signifikanten Verbesserung des ACR CRISS-Scores, sowie einer numerischen Reduktion des FVC-Verlustes bei guter Verträglichkeit [27].

\section{Rituximab}

Rituximab ist ein B-Zell-depletierender Antikörper, der bei vielen Autoimmun- erkrankungen und bei B-Zell-Lymphomen eingesetzt wird. In einer offenen, randomisierten Studie war Rituximab (2mal $1000 \mathrm{mg}$ intravenös, im Abstand von 2 Wochen) bei SSc-ILD gegenüber CYC $\left(500 \mathrm{mg} / \mathrm{m}^{2}\right.$ alle 4 Wochen) nach 6 Monaten in Bezug auf die Verbesserung der FVC überlegen und verursachte zudem weniger schwere unerwünschte Ereignisse [40].

In einer Single-Center-Studie führte eine Kombinationstherapie aus Rituximab (14-tägig $1000 \mathrm{mg} \times 2$ oder $375 \mathrm{mg} / \mathrm{m}^{2} /$ Woche für 4 aufeinanderfolgende Wochen) und MMF (2000 mg/Tag) zu einer signifikanten Verbesserung des modifizierten Rodnan Skin Scores (mRSS) und der ILD-Ausdehnung in der Lunge [12].

\section{Riociguat}

Riociguat, ein oral wirksamer Stimulator des Enzyms lösliche Guanylatcyclase (sGC), erwies sich zur Therapie der pulmonalen Hypertonie als vorteilhaft [14].

In RISE-SSc, einer randomisierten Phase-IIb-Studie, wurden die Wirksamkeit und Sicherheit von Riociguat bei SSc-Patienten untersucht [9]. Der primäre Endpunkt der Studie, die absolute Veränderung des modifizierten Rodnan Skin Scores (mRSS) gegenüber dem Ausgangswert nach 52 Wochen, wurde trotz eines Trends nicht erreicht. Riociguat stabilisierte jedoch die Lungenfunktion vs. Placebo und war mit einer guten Verträglichkeit assoziiert [7].

\section{Antifibrotika}

Die Pathophysiologie der SSc-ILD zeigt Gemeinsamkeiten mit der Entstehung anderer fibrotischer Erkrankungen wie etwa der idiopathischen Lungenfibrose (IPF), woraus sich mögliche Optionen für die Entwicklung neuer Behandlungsansätze der SSc-ILD ergeben.

\section{Pirfenidon}

Pirfenidon ist ein oral zu verabreichender Wirkstoff aus der Gruppe der Antifibrotika, dessen Wirksamkeit bei Betroffenen mit IPF in den placebokontrollierten CAPACITY- und ASCEND-Phase- 
II- und -III-Studien erfolgreich gezeigt wurde [28, 33].

Die LOTUSS-Studie, eine randomisierte Phase-II-Studie, in die 63 Patienten mit SSc-ILD eingeschlossen wurden, zielte darauf ab, die Verträglichkeit und Sicherheit von Pirfenidon bei Patienten mit SSc-ILD zu bewerten. Unter der Pirfenidon-Therapie kam es gehäuft zu Übelkeit, Kopfschmerzen und Müdigkeit [24]. Hinsichtlich des Abfalles der FVC konnte kein signifikanter Unterschied zwischen beiden Studienarmen gezeigt werden, die untersuchte Patientenanzahl war allerdings zu klein, um die Wirksamkeit von Pirfenidon abschließend zu beurteilen.

RELIEF, eine randomisierte, doppelblinde, placebokontrollierte Studie des Deutschen Zentrums für Lungenforschung (DZL), untersuchte die Wirkung von Pirfenidon auf diverse Nicht-IPFLungenfibroseformen, u.a. auch SScILD [3]. Die Ergebnisse wurden auf dem European Respiratory Society Congress vorgestellt und zeigten einen signifikant niedrigeren Abfall der FVC unter Pirfenidontherapie.

Eine weitere randomisierte PhaseII-Studie, die Scleroderma Lung Study (SLS III), untersucht zurzeit den Einfluss einer Kombinationstherapie aus Mycophenolat-Mofetil (MMF) und Pirfenidon auf den Verlauf der Lungenfunktion bei Patienten mit SSc-ILD. Erste Studienergebnisse der SLS III-Studie werden für das Jahr 2021 erwartet.

\section{Nintedanib}

Nintedanib, ein oral zu verabreichender Multityrosinkinaseinhibitor, hemmt über die Rezeptoren für FGF, VEGF, PDGF und TGF- $\beta$ wichtige, für die SScILD relevante Pathomechanismen [50]. Die Wirksamkeit und Sicherheit von Nintedanib bei IPF wurde in 3 PhaseII- und -III-Studien, TOMORROW, INPULSIS $^{\circledR}-1$ und INPULSIS ${ }^{\circledR}-2$, erfolgreich gezeigt $[36,37]$. Dabei senkte Nintedanib die jährliche Abnahme der Vitalkapazität (FVC) gegenüber Placebo signifikant [37]. Nintedanib wies ein akzeptables Nebenwirkungsprofil auf, mit Diarrhö als häufigste Nebenwirkung [37].

In präklinischen Studien mit unterschiedlichen Mausmodellen zeigte
Nintedanib potente antifibrotische Effekte $[20,21]$. Im transgenen Fra2Mausmodell ließen sich auch positive Effekte auf die SSc-artigen vaskulären Veränderungen nachweisen [20, 21].

Angesichts dieser Ergebnisse untersuchen derzeit 2 groß angelegte klinische Phase-III-Studien den Effekt von Nintedanib bei SSc-ILD und progredient fibrosierender ILD (PF-ILD) [49].

Die randomisierte, doppelblinde, placebokontrollierte Phase-III-Studie SENSCIS $^{\circledR}$ untersuchte Wirksamkeit und Sicherheit von Nintedanib bei Patienten mit SSc-ILD [8]. Vergleichbar mit den Ergebnissen der INPULSIS ${ }^{\circledR}$-Studien bei IPF reduzierte Nintedanib am Ende der 52-wöchigen Studie auch bei Patienten mit SSc-ILD die jährliche Rate des FVC-Abfalls signifikant um $44 \%$ vs. Placebo. Der adjustierte jährliche FVCVerlust bei Patienten, die mit Nintedanib behandelt wurden, betrug $-52,4 \mathrm{ml} /$ Jahr im Vergleich zu -93,3 ml/Jahr mit Placebo (absolute Differenz 41,0 ml/Jahr [95\%-KI: 2,9-79,0; $p=0,035]$ ). In die Studie wurde ein breites Spektrum von SSc-ILD-Patienten eingeschlossen, darunter auch rund $50 \%$ Patienten mit Mycophenolat-Mofetil (MMF) als Begleitmedikation zu Studienbeginn. Die relative Reduktion der FVC betrug mit MMF $40 \%$ und ohne MMF $46 \%$ (Interaktions- $p$-Wert: $p=0,452)$. Es zeigte sich eine numerische additive Wirksamkeit der Nintedanib und MycophenolatTherapie. Ein Effekt von Nintedanib auf die Hautfibrose oder Lebensqualität wurde nicht beobachtet. Das Sicherheits- und Verträglichkeitsprofil waren akzeptabel und vergleichbar mit dem Nebenwirkungsprofil bei IPF-Patienten [8].

Die doppelblinde, randomisierte, placebokontrollierte Phase-III-Studie INBUILD $^{\circledR}$ untersuchte die Wirksamkeit und Sicherheit von Nintedanib über 52 Wochen bei Patienten mit progredient fibrosierender interstitieller Lungenerkrankung (PF-ILD) unabhängig von ihrer Grunderkrankung mit Ausnahme der idiopathischen Lungenfibrose (IPF) (• Abb. 3; [11]). Die adjustierte jährliche Abnahme der FVC über 52 Wochen betrug in der gesamten Studienpopulation $-80,8 \mathrm{ml} / \mathrm{Jahr}$, verglichen $\mathrm{mit}-187,8 \mathrm{ml} /$
Jahr für Placebo (Differenz: 107,0 ml/ Jahr [95\%-KI: 65,4-148,5], $p<0,001$ ) [11].

\section{Ausblick}

Die interstitielle Lungenbeteiligung ist eine häufige Organmanifestation der systemischen Sklerose und geht mit einem erhöhten Morbiditäts- und Mortalitätsrisiko einher. Aufgrund zahlreicher neuer Studiendaten unterliegen die Therapiekonzepte für die SSc-assoziierte ILD aktuell einem Wandel. Die Vermeidung von Risikofaktoren wie Zigarettenrauchen, Staubbelastung und Mikroaspirationen sind wichtige Bausteine der Behandlung. Die derzeitig verfügbaren medikamentösen Therapieansätze zielen darauf $a b$, über immunmodulierende und antiinflammatorische Wirkung die Krankheitsprogression zu verlangsamen. Die aktuelle EULAR-Leitlinie empfiehlt den Einsatz von Cyclophosphamid und HSCT. Als weitere neue immunmodulatorische Therapieoptionen werden Mycophenolat und Tocilizumab in klinischen Studien weiter untersucht. Unter Mycophenolat-Therapie wurde der jährliche FVC-Abfall reduziert. Aktuell werden die antifibrotischen Wirkstoffe Nintedanib und Pirfenidon in mehreren klinischen Studien zur Behandlung der SSc-ILD getestet. Nintedanib konnte hier signifikant das Fortschreiten der Erkrankung bremsen und hat einen numerischen additiven Effekt zur Mycophenolat-Therapie. Diese positiven Daten bilden die Grundlage für den $\mathrm{Zu}$ lassungsantrag für Nintedanib bei $\mathrm{SSc}$ ILD. In den USA ist Nintedanib bei SScILD seit September 2019 von der FDA zugelassen.

Erfolg versprechende therapeutische Perspektiven für die SSc-ILD sind somit durch die Ansätze aktueller und bevorstehender Arzneimittelstudien gegeben und werden in Zukunft die Prognose von SScILD Betroffenen merklich verbessern.

\section{Fazit für die Praxis}

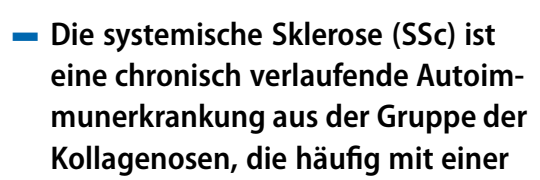




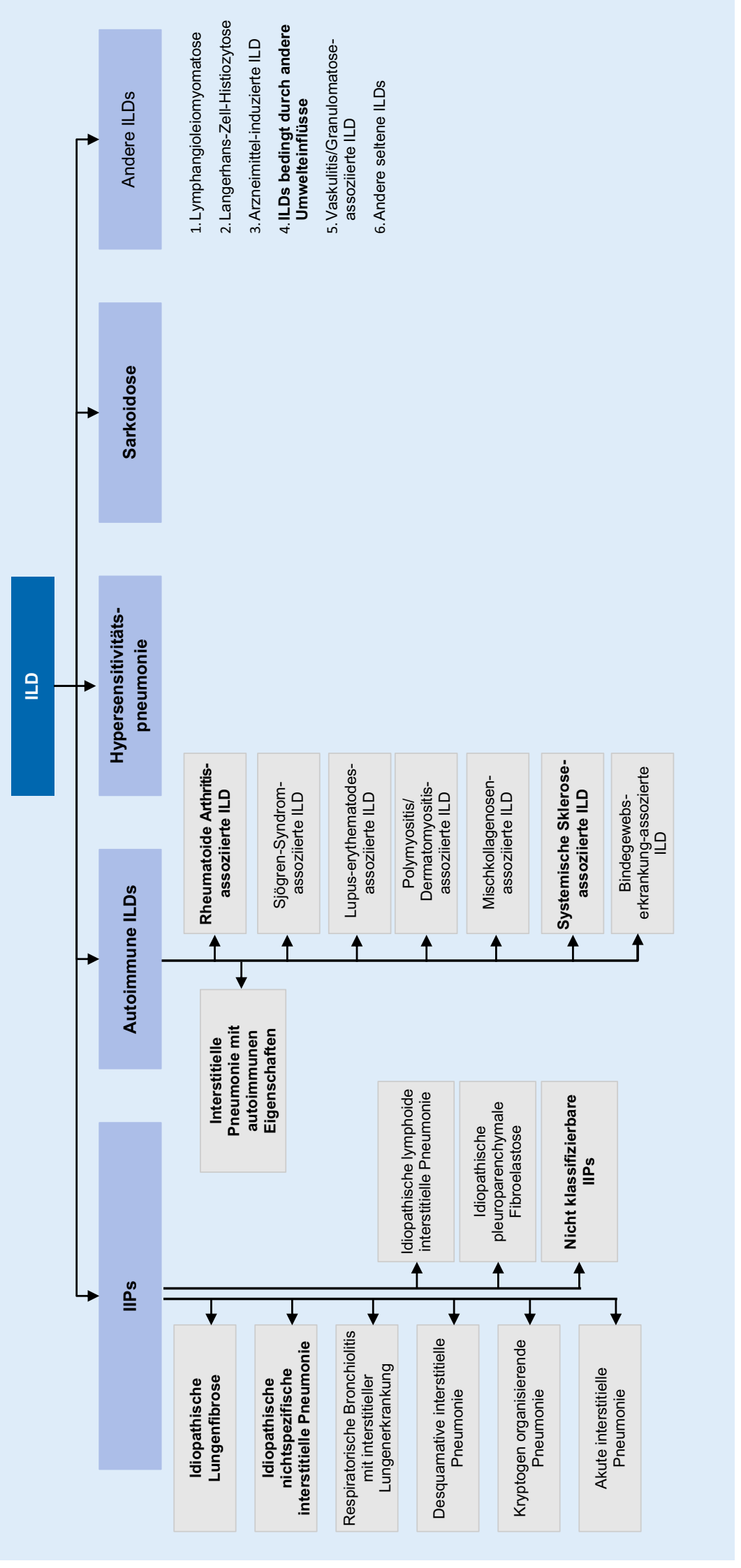

pulmonalen Beteiligung in Form einer ILD einhergeht.

- Bisher gibt es nur eingeschränkte Therapiemöglichkeiten zur Behandlung der SSC-ILD.

- Eine Reihe klinischer Studien untersucht jedoch derzeit vielversprechende Pharmakotherapien zur Behandlung der Lungenfibrose bei SSc, darunter Tocilizumab und Anabasum.

- Aufgrund der pathophysiologischen Gemeinsamkeiten zu anderen fibrotischen Lungenerkrankungen kann der antifibrotische Wirkstoff Nintedanib ein Erfolg versprechender zusätzlicher Behandlungsansatz sein, was kürzlich bereits durch erste positive Studienergebnisse belegt wurde.

\section{Korrespondenzadresse}

Prof. Dr. A. Prasse
Klinik für Pneumologie,
Medizinische Hochschule
Hannover
Carl-Neuberg-Str. 1,
30625 Hannover,
Deutschland
Prasse.Antje@mh-
hannover.de

Danksagung. Die vorliegende Publikation wurde durch die finanzielle Unterstützung der Firma Boehringer Ingelheim Pharma GmbH \& Co. KG ermöglicht. Redaktionelle Unterstützung und Medical Writing wurden während der Vorbereitung und Erstellung dieses Artikels von Anna Hermann, Firma infill healthcare communication $\mathrm{GmbH}$, bereitgestellt. Die auf der Titelseite des Manuskripts aufgeführten Autoren haben alle wesentliche Beiträge geleistet, die sie als Autoren qualifizieren. Alle Autoren haben die eingereichte Version endgültig zur Veröffentlichung freigegeben.

\section{Einhaltung ethischer Richtlinien}

Interessenkonflikt. A. Prasse: Berater- und Vortragshonorare, Reisekostenübernahme von Boehringer Ingelheim, Roche, Novartis, AbbVie, Pliant und AstraZeneca. F. Bonella: Berater-und Vortragshonorare, Reisekostenübernahme sowie Sachmittelunterstützung von Boehringer Ingelheim. U. Müller-Ladner: Berater- und Vortragshonorare von Boehringer Ingelheim. T. Witte: Berater- und Vortragshonorare von Boehringer Ingelheim. N. Hunzelmann: Berater- und Vortragshonorare von Actelion, Boehringer Ingelheim und Roche. J. Distler: Berater- und Vortragshonorare sowie Sachmittelunterstützung von Boehringer Ingelheim.

Abb. 3 ॥ ILDs, die einen progredient fibrosierenden Phänotypen entwickeln können [1,5] 
Für diesen Beitrag wurden von den Autoren keine Studien an Menschen oder Tieren durchgeführt. Für die aufgeführten Studien gelten die jeweils dort angegebenen ethischen Richtlinien.

Open Access. Dieser Artikel wird unter der Creative Commons Namensnennung 4.0 International Lizenz (http://creativecommons.org/licenses/by/4.0/deed de) veröffentlicht, welche die Nutzung, Vervielfältigung, Bearbeitung, Verbreitung und Wiedergabe in jeglichem Medium und Format erlaubt, sofern Sie den/die ursprünglichen Autor(en) und die Quelle ordnungsgemäßnennen, einen Linkzur Creative Commons Lizenz beifügen und angeben, ob Änderungen vorgenommen wurden.

\section{Literatur}

1. American Thoracic Society, European Respiratory Society (2002) American Thoracic Society/ European Respiratory Society international multidisciplinary consensus classification of the idiopathic interstitial pneumonias. Am J Respir Crit Care Med 165:277-304. https://doi.org/10.1164/ ajrccm.165.2.ats01

2. Assassi S, SharifR, Lasky RE etal (2010) Predictors of interstitial lung disease in early systemic sclerosis: a prospective longitudinal study of the GENISOS cohort. Arthritis Res Ther 12:R166. https://doi.org/ 10.1186/ar3125

3. Behr J, Neuser P, Prasse A et al (2017) Exploring efficacy and safety of oral Pirfenidone for progressive non-IPF lung fibrosis (RELIEF) - a randomized, double-blind, placebo-controlled, parallel group, multi-center, phase II trial. BMC Pulm Med 17:122. https://doi.org/10.1186/s12890-017-0462-y

4. Chowaniec M, Skoczyńska M, Sokolik R, Wiland P (2018) Interstitial lung disease in systemic sclerosis: challenges in early diagnosis and management. Reumatologia 56:249-254. https://doi.org/10. 5114/reum.2018.77977

5. Cottin V, Hirani NA, Hotchkin DL et al (2018) Presentation, diagnosis and clinical course of the spectrum of progressive-fibrosing interstitial lung diseases. Eur Respir Rev. https://doi.org/10.1183/ 16000617.0076-2018

6. Denton CP, Khanna D (2017) Systemic sclerosis. Lancet 390:1685-1699. https://doi.org/10.1016/ S0140-6736(17)30933-9

7. Distler O, Allanore Y, Denton CP et al (2018) Riociguat in patients with early diffuse cutaneous systemic sclerosis: a randomized, double-blind placebo-controlled phase IIb study (RISE-Ssc) [abstract]. Arthritis Rheumatol 70(suppl 10): https://acrabstracts.org/abstract/riociguat-in patients-with-early-diffuse-cutaneous-systemicsclerosis-a-randomized-double-blind-placebocontrolled-phase-iib-study-rise-ssc/. Accessed November 13,2019

8. Distler O, Highland KB, Gahlemann M et al (2019) Nintedanib for systemic sclerosis-associated interstitial lung disease. N Engl J Med. https://doi. org/10.1056/NEJMoa1903076

9. Distler O, Pope J, Denton C et al (2017) RISE-SSc: riociguat in diffuse cutaneous systemic sclerosis. Respir Med 122(Suppl 1):S14-S17.https://doi.org/ 10.1016/j.rmed.2016.09.011

10. Domsic RT (2014) Scleroderma: the role of serum autoantibodies in defining specific clinical phenotypes and organ system involvement. Curr Opin Rheumatol 26:646-652. https://doi.org/10. 1097/BOR.0000000000000113
11. Flaherty KR, Wells AU, Cottin V et al (2019) Nintedanib in progressive fibrosing interstitial lung diseases. N Engl J Med. https://doi.org/10. 1056/NEJMoa1908681

12. Fraticelli P, Fischetti C, Salaffi F et al (2018) Combination therapy with rituximab and mycophenolate mofetil in systemic sclerosis. A single-centre case series study. Clin Exp Rheumatol 36(Suppl 113):142-145

13. Gabrielli A, Avvedimento EV, Krieg T (2009) Scleroderma. N Engl J Med 360:1989-2003. https://doi.org/10.1056/NEJMra0806188

14. Ghofrani H-A, Galiè N, Grimminger $F$ et al (2013) Riociguat for the treatment of pulmonary arteria hypertension. N Engl J Med 369:330-340. https:// doi.org/10.1056/NEJMoa1209655

15. Giacomelli R, Liakouli V, Berardicurti O et al (2017) Interstitial lung disease in systemic sclerosis: current and future treatment. Rheumatol Int 37:853-863. https://doi.org/10.1007/s00296016-3636-7

16. Goh NSL, Desai SR, Veeraraghavan S et al (2008) Interstitial lung disease in systemic sclerosis: a simple staging system. Am J Respir Crit Care Med 177:1248-1254. https://doi.org/10.1164/rccm. 200706-8770C

17. Hamaguchi Y (2010) Autoantibody profiles in systemic sclerosis: predictive value for clinical evaluation and prognosis. J Dermatol 37:42-53. https://doi.org/10.1111/j.1346-8138.2009.00762. $x$

18. van den Hoogen F, Khanna D, Fransen J et al (2013) 2013 classification criteria for systemic sclerosis: an American College of Rheumatology/European League against Rheumatism collaborative initiative. Arthritis Rheum 65:2737-2747. https://doi. org/10.1002/art.38098

19. Hoyles RK, Ellis RW, Wellsbury J et al (2006) A multicenter, prospective, randomized, doubleblind, placebo-controlled trial of corticosteroids and intravenous cyclophosphamide followed by oral azathioprine for the treatment of pulmonary fibrosis in scleroderma. Arthritis Rheum 54:3962-3970. https://doi.org/10.1002/art.22204

20. Huang J, Beyer C, Palumbo-Zerr K et al (2016) Nintedanib inhibits fibroblast activation and ame liorates fibrosis in preclinical models of systemic sclerosis. Ann Rheum Dis 75:883-890. https://doi. org/10.1136/annrheumdis-2014-207109

21. Huang J, Maier C, Zhang Y et al (2017) Nintedanib inhibits macrophage activation and ameliorates vascular and fibrotic manifestations in the Fra2 mouse model of systemic sclerosis. Ann Rheum Dis 76:1941-1948. https://doi.org/10. 1136/annrheumdis-2016-210823

22. Hunzelmann N, Genth E, Krieg T et al (2008) The registry of the German Network for Systemic Scleroderma: frequency of disease subsets and patterns of organ involvement. Rheumatology 47:1185-1192. https://doi.org/10.1093/ rheumatology/ken179

23. Jaeger VK, Wirz EG, Allanore $Y$ et al (2016) Incidences and risk factors of organ manifestations in the early course of systemic sclerosis: a longitudinal EUSTAR study. PLOS ONE 11:e163894. https://doi.org/10.1371/journal.pone.0163894

24. Khanna D, Albera C, Fischer A et al (2016) An openlabel, phase II study of the safety and tolerability of pirfenidone in patients with sclerodermaassociated interstitial lung disease: the LOTUSS trial. J Rheumatol 43:1672-1679. https://doi.org/ 10.3899/jrheum.151322

25. Khanna D, Denton CP, Lin CJF etal (2018) Safetyand efficacy of subcutaneous tocilizumab in systemic sclerosis: results from the open-label period of a phase II randomised controlled trial (faSScinate). Ann Rheum Dis 77:212-220. https://doi.org/10. 1136/annrheumdis-2017-211682

26. Khanna D, Lin CJF, Kuwana M et al (2018) Efficacy and safety of tocilizumab for the treatment of systemic sclerosis: results from a phase 3 randomized controlled trial [abstract]. Arthritis Rheumatol 70(suppl 10): https://acrabstracts. org/abstract/efficacy-and-safety-of-tocilizumabfor-the-treatment-of-systemic-sclerosis-resultsfrom-a-phase-3-randomized-controlled-trial/. Accessed November 13, 2019

27. Khanna D, Spino C, Bush E et al (2018) Abatacept vs. Placebo in early diffuse cutaneous systemic sclerosis-results of a phase 2 investigator initiated, double-blind, placebo-controlled, multicenter, randomized controlled trial study [abstract]. Arthritis Rheumatol 70(suppl 10): https://acrabstracts. org/abstract/abatacept-vs-placebo-in-earlydiffuse-cutaneous-systemic-sclerosis-results-ofa-phase-2-investigator-initiated-double-blindplacebo-controlled-multicenter-randomizedcontrolled-trial-stu/. Accessed November 13, 2019

28. King TE, Bradford WZ, Castro-Bernardini $S$ et al (2014) A phase 3 trial of pirfenidone in patients with idiopathic pulmonary fibrosis. N Engl J Med 370:2083-2092. https://doi.org/10.1056/ NEJMoa1402582

29. Kowal-Bielecka O, Fransen J, Avouac J et al (2017) Update of EULAR recommendations for the treatment of systemic sclerosis. Ann Rheum Dis 76:1327-1339. https://doi.org/10. 1136/annrheumdis-2016-209909

30. van Laar JM, FargeD, Sont JKetal (2014) Autologous hematopoietic stem cell transplantation vs intravenous pulse cyclophosphamide in diffuse cutaneous systemic sclerosis: a randomized clinical trial. JAMA 311:2490-2498. https://doi.org/10. 1001/jama.2014.6368

31. Nadashkevich O, Davis P, Fritzler M, Kovalenko W (2006) A randomized unblinded trial of cyclophosphamide versus azathioprine in the treatment of systemic sclerosis. Clin Rheumatol 25:205-212. https://doi.org/10.1007/s10067-005-1157-y

32. Nihtyanova SI, Denton CP (2010) Autoantibodies as predictive tools in systemic sclerosis. Nat Rev Rheumatol 6:112-116. https://doi.org/10.1038/ nrrheum.2009.238

33. Noble PW, Albera C, Bradford WZ et al (2011) Pirfenidone in patients with idiopathic pulmonary fibrosis (CAPACITY): two randomised trials. Lancet 377:1760-1769. https://doi.org/10.1016/S01406736(11)60405-4

34. Raghu G, Collard HR, Egan JJ et al (2011) An official ATS/ERS/JRS/ALAT statement: idiopathic pulmonary fibrosis: evidence-based guidelines for diagnosis and management. Am J Respir Crit Care Med 183:788-824. https://doi.org/10.1164/rccm. 2009-040GL

35. Rahaghi F, Strek M, Southern B et al (2019) Expert Consensus on the Screening, Treatment, and Management of Patients with Systemic Sclerosis-Associated Interstitial Lung Disease (SSc-ILD), and the Potential Future Role of Anti-fibrotics[asterisk] in a Treatment Paradigm for SSC-ILD: A Delphi Consensus Study. C101 NON-IDIOPATHIC INTERSTITIAL PNEUMONIA Nat Hist Progn ILD Ther:A7445-A7445. https:// doi.org/10.1164/ajrccm-conference.2019.199.1_ MeetingAbstracts.A7445

36. Richeldi L, Costabel U, Selman M et al (2011) Efficacy of a tyrosine kinase inhibitor in idiopathic 
pulmonary fibrosis. N Engl J Med 365:1079-1087. https://doi.org/10.1056/NEJMoa1103690

37. Richeldi L, Du Bois RM, Raghu G (2014) Efficacy and safety of nintedanib in idiopathic pulmonary fibrosis. N Engl J Med 370:2071-2082. https://doi. org/10.1056/NEJMx150012

38. Schupp J, Günther J, Müller-Quernheim J et al (2014) Serum CCL18 is predictive for lung disease progression and mortality in systemic sclerosis European Respiratory Society. Eur Respir J 43:1529-1530

39. SilverKC, Silver RM (2015) Management of systemic sclerosis-associated interstitial lung disease (SscILD). Rheum Dis Clin NorthAm41:439-457.https:// doi.org/10.1016/j.rdc.2015.04.006

40. Sircar G, Goswami RP, Sircar D et al (2018) Intravenous cyclophosphamide vs rituximab for the treatment of early diffuse scleroderma lung disease: open label, randomized, controlled trial. Rheumatology 57:2106-2113. https://doi.org/10. 1093/rheumatology/key213

41. Spiera RF, Hummers L, Chung L et al (2017) A phase 2 study of safety and efficacy of anabasum (JBT-101), a cannabinoid receptor type 2 agonist, in diffuse cutaneous systemic sclerosis [abstract]. Arthritis Rheumatol 69(suppl 10): https://acrabstracts.org/abstract/a-phase-2study-of-safety-and-efficacy-of-anabasum-jbt101-a-cannabinoid-receptor-type-2-agonist-indiffuse-cutaneous-systemic-sclerosis/. Accessed November 13, 2019

42. Steen VD, Medsger TA (2007) Changes in causes of death in systemic sclerosis, 1972-2002. Ann Rheum Dis 66:940-944. https://doi.org/10.1136/ ard.2006.066068

43. Sullivan KM, Goldmuntz EA, Keyes-Elstein L et al (2018) Myeloablative autologous stem-cell transplantation for severe scleroderma. N Engl J Med 378:35-47
44. Tashkin DP, Elashoff R, Clements PJ et al (2006) Cyclophosphamide versus placebo in scleroderma lung disease. NEngl J Med 354:2655-2666. https:// doi.org/10.1056/NEJMoa055120

45. Tashkin DP, Roth MD, Clements PJetal (2016) Mycophenolate mofetil versus oral cyclophosphamide in scleroderma-related interstitial lung disease (SLS II): a randomised controlled, double-blind, parallel group trial. Lancet Respir Med 4:708-719. https://doi.org/10.1016/S2213-2600(16)30152-7

46. Tyndall AJ, Bannert B, Vonk M et al (2010) Causes and risk factors for death in systemic sclerosis: a study from the EULAR Scleroderma Trials and Research (EUSTAR) database. Ann Rheum Dis 69:1809-1815. https://doi.org/10.1136/ard.2009. 114264

47. Usategui A, del Rey MJ, Pablos JL (2011) Fibroblast abnormalities in the pathogenesis of systemic sclerosis. Expert Rev Clin Immunol 7:491-498. https://doi.org/10.1586/eci.11.39

48. Volkmann ER, Tashkin DP (2016) Treatment of systemic sclerosis-related interstitial lung disease: a review of existing and emerging therapies. Ann Am Thorac Soc 13:2045-2056. https://doi.org/10. 1513/AnnalsATS.201606-426FR

49. Wollin L, Distler JH, Denton CP, Gahlemann M (2019) Rationale for the evaluation of nintedanib as a treatment for systemic sclerosis-associated interstitial lung disease.JScleroderma RelatDisord. https://doi.org/10.1177/2397198319841842

50. Wollin L, Wex E, Pautsch A et al (2015) Mode of action of nintedanib in the treatment of idiopathic pulmonary fibrosis. Eur Respir J 45:1434-1445. https://doi.org/10.1183/09031936.00174914

51. Wu W, Jordan S, Graf N et al (2019) Progressive skin fibrosis is associated with a decline in lung function and worse survival in patients with diffuse cutaneous systemic sclerosis in the European Scleroderma Trials and Research
(EUSTAR) cohort. Ann Rheum Dis. https://doi.org/ 10.1136/annrheumdis-2018-213455

52. Xanthouli P, Hermann W, Hunzelmann N, Kreuter M (2018) Sklerodermie-assoziierte interstitielle Lungenerkrankungen. Pneumologe 15:383-395. https://doi.org/10.1007/s10405-018-0204-8

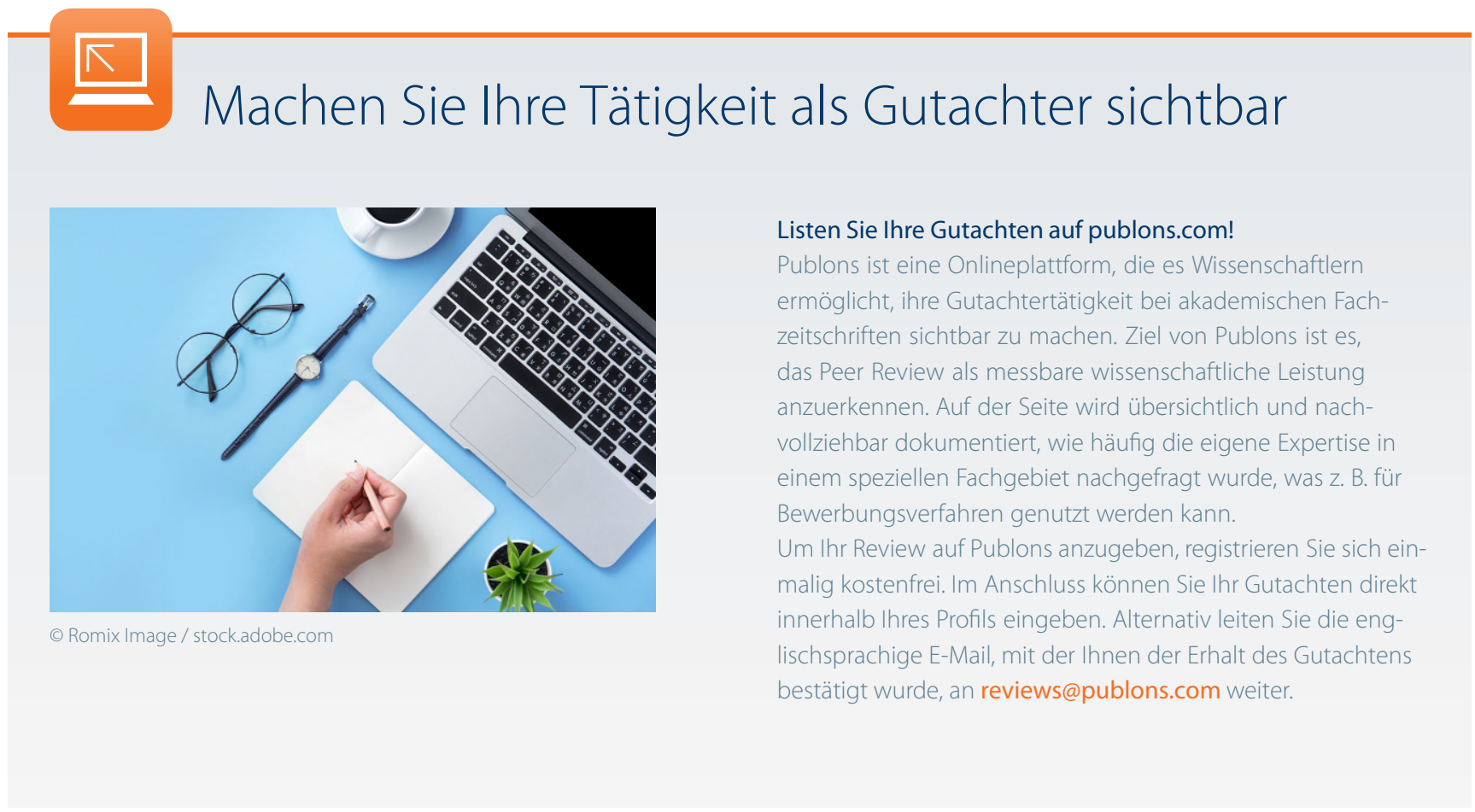

